\title{
Design of a Fuzzy Networked Control Systems. Priority Exchange Scheduling Algorithm
}

\author{
H. Benítez-Pérez, J. Ortega-Arjona, J.A. Rojas-Vargas, A. Durán-Chavesti
}

\author{
H. Benítez-Pérez \\ Universidad Nacional Autónoma de México \\ Apdo. Postal 20-726, Admón. 20, Del. A. Obregón, México D. F., CP. 01000. \\ hector.benitez@iimas.unam.mx
}

\section{Jorge Ortega-Arjona}

Facultad de Ciencias UNAM

Av. Universidad 3000, C. U., México D. F.

jloa@ciencias.unam.mx

Jared A. Rojas-Vargas

IIMAS UNAM

Cto. Escolar 3000, C. U., México D. F.

jared_36_23@hotmail.com

\section{A. Durán-Chavesti*}

Universidad Nacional Autónoma de México

Apdo. Postal 20-726, Admón. 20, Del. A. Obregón, México D. F., CP. 01000.

*Corresponding author: adrian.chavesti@iimas.unam.mx

\begin{abstract}
This work presents a supervisory control strategy for Networked Control Systems (NCSs). This shows the identification and control of the plant using fuzzy theory. The fuzzy model incorporates the delay dynamics within the fuzzy rules based upon a real-time hierarchical scheduling strategy. A hierarchical scheduling Priority Exchange algorithm is used based upon codesign strategy following mutual correlation among control and network algorithms in order to bounded time delays. A system of magnetic levitation is presented as a case study.

Keywords: Fuzzy control, networked control system, time delay codesign.
\end{abstract}

\section{Introduction}

The control design and stability analysis of network-based control systems (NCSs) have been studied in recent years [14], [8] and [24] based upon codesign strategy. The main advantages of this kind of systems are their low cost, small volume of wiring, distributed processing, simple installation, maintenance and reliability.

In a NCS, one of the key issue is the effect of network-induced delay in the system performance. The delay can be constant, time-varying, or even random, this depends on the scheduler, network type, architecture, operating systems, etc [24]. One strategy to be followed is the codesign since it takes both desired procedures to be followed. Nilsson analyzes several important facets of NCSs [15]. It introduces models for the delays in NCS, first as a fixed delay, after as an independently random, and finally like a Markov process. The author introduces optimal stochastic control theorems for NCSs based upon the independently random and Markovian delay models. In [18], introduces static and dynamic scheduling policies for transmission of sensor data in a continuous-time LTI system. They introduce the notion of the maximum allowable transfer interval (MATI), which is the longest time after a sensor should transmit a data. [18] derived bounds of the MATI such that the NCS is stable. This MATI ensures that the Lyapunov function of the system under consideration is strictly decreasing at all times. In [22] extends the 
work of Walsh., he developed a theorem which ensures the decrease of a Lyapunov function for a discrete-time LTI system at each sampling instant, using two different bounds. These results are less conservative than those of Walsh, because he doesn't require the system's Lyapunov function to be strictly decreasing at all time.

Besides, following the work presented by [13] although the strategy is similar as well as the case study in here, the proposed fuzzy control follows each local time delay produced by the scheduling algorithm which is dynamic and reactive to external tasks modification (as Priority Exchange Proposes). Although the results are stable in both cases, in here the challenging strategy is to dismiss dynamic local time delays without forcing system bounds. It is important to mention that this work follows the expressions designed in [3], [4] and [3] with the characteristic of real local time delays and local gain control design following eqn. 10 and LMI procedure as presented in section 4. In [7], [17], [20] and [21] introduce a number of different linear matrix inequality (LMI) tools for analyzing and designing optimal switched NCSs. [23] takes into consideration both the network-induced delay and the time delay the plant, a controller design method is proposed by using the delay-dependent approach. An appropriate Lyapunov functional candidate is utilized to obtain a memoryless feedback controller, this is derived by solving a set of Linear Matrix Inequalities (LMIs). In [19] models the network induced delays of the NCSs as interval variables governed by a Markov chain. Using the upper and lower bounds of the delays, a discrete-time Markovian jump system with norm-bounded uncertainties is presented to model the NCSs. Based on this model, the $\mathrm{H} \infty$ state feedback controller can be constructed via a set of LMIs. Recently [9] introduced a new (descriptor) model transformation for delay-dependent stability for systems with time-varying delays in terms of LMIs, and she also refines recent results on delay-dependent $\mathrm{H} \infty$ control and extend them to the case of time-varying delays.

Alternatively [10] takes into consideration both the network-induced delay and the time delay in the plant, and thus, introduces a controller design method, using the delay-dependent approach. An appropriate Lyapunov functional candidate is used to obtain a memoryless feedback controller, derived by solving a set of Linear Matrix Inequalities (LMIs) [6]. [11] models the network induced delays of the NCSs as interval variables governed by a Markov chain. Using the upper and lower bounds of the delays, a discrete-time Markovian jump system with norm-bounded uncertainties is presented to model the NCSs. Based on this model, a $\mathrm{H} \infty$ state feedback controller can be constructed via a set of LMIs.

An interesting approximation has been presented by [2] where time delays incorporation has been proposed following state space representation.

\section{Systems Proposal}

Based on this review, this paper defines a model (Fig. 1) that integrates the time delays for a class of nonlinear system, where the actual proposal it is the enhancement of states in order to represent of control and plant states to fulfill a complete modeling of time delays according to priority exchange Dynamic Schedulling Algorithm.

It comprises two types of fuzzy rules, one that models the dynamics of the plant and another that introduces the networked-induced time delay. It involves estimating the time delay based scheduling behaviour where the fuzzy rules are such as:

$$
\begin{gathered}
\text { if } x_{i}(k) \text { is } \mu_{i j} \text { then } x_{j}^{N}(k+1)=A_{j} x(k)+B_{0} u(k) \\
i=1 \ldots n j=1 \ldots r h=1 \ldots s
\end{gathered}
$$




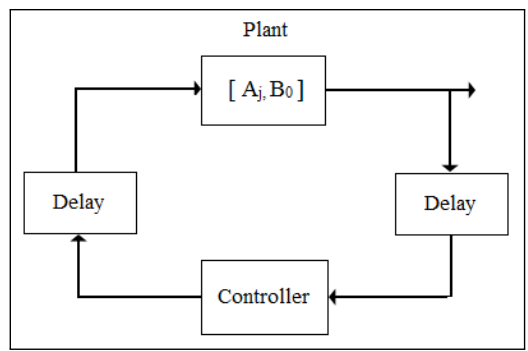

Figure 1: Fuzzy model proposed.

The overall system is:

$$
\widehat{x}(k+1)=\sum_{j=1}^{r} x_{j}^{N}+\sum_{h=1}^{s} x_{h}^{D}=\sum_{j=1}^{r} R_{j} A_{j} x(k)+\sum_{h=1}^{s} S_{h}\left(B_{0}\right) u(k)
$$

where $x_{i}$ is the $i$ th state of the plant, $\mu_{i j}$ is the membership function of the $i t h$ state and $s$ is the total number of local time delays and the $j$ th rule. $A_{j} \in R^{n x n}, B_{0, h} \in R^{n}, x \in R^{n}, u(k)$ $\in R$, with $n$ states, and $r$ nominal fuzzy rules, sh is he nominal selection of current fuzzy rule. Where $N$ and $D$ denote nominal and delayed model respectively. The fire strength $\psi_{j}$ is defined as the function multiplication between the membership functions $\mu_{i j}$.

$$
\begin{gathered}
\mu_{i j}=\exp \left(-\frac{\left(x_{i}-c_{i j}\right)^{2}}{\sigma_{i j}^{2}}\right) \\
\psi_{j}=\prod_{i=1}^{m} \mu_{i j} \\
R_{j}=\frac{\psi_{j}}{\sum_{k=1}^{r} \psi_{k}} \\
0<R_{j} \leq 1, \sum_{j=1}^{r} R_{j}(x)=1
\end{gathered}
$$

For the s fuzzy rules with delay $\tau_{c a h}, \nu_{h}$ is the gaussian membership function of the time delay with center $\alpha_{h}$ and standard deviation $\beta_{h}$.

$$
\begin{gathered}
S_{h}=\frac{\nu_{h}}{\sum_{k=1}^{s} \nu_{k}} \\
\nu_{h}=\exp \left(-\frac{\left(\tau_{c a h}-\alpha_{h}\right)^{2}}{\beta_{h}^{2}}\right)
\end{gathered}
$$

The proposed decomposition in terms of feedback state space representation has been reviewed by [4], where the indexing is defined by the time delays as local and bounded situations through the network.

Firstly, as augmented states and the related bounded time delays of plant and controller, following the strategies presented in [3], [4] and [3] the control structure is modified according to a particular gain control per local time delays scenarios and different local operational points from a particular case study. In here the strategy is modified by designing local control laws as 
gain rather them a dynamic state feedback control.

The results as shown in section 5 are quite promising in that respect, it is presented as such:

$$
\begin{gathered}
X=\left[\begin{array}{l}
x_{c} \\
x_{p}
\end{array}\right] \\
x_{c}(k+1)=\sum_{j=1}^{N} \sum_{i=1}^{N}\left[h_{j} h_{i}\left[B_{j}^{p}\left(x_{c}\left(k-t_{c a i}\right)\right)\right]+h_{j} A_{j}^{p} x_{p}(k)\right] \\
x_{p}(k+1)=\begin{array}{c}
\sum_{j=1}^{N} \sum_{i=1}^{N}\left[h_{j} h_{i}\left[F_{j}^{c}\left(c_{p}^{i} x_{p}\left(k-t_{s c i}\right)\right)\right]+h_{j} F_{j}^{c}\right]
\end{array}
\end{gathered}
$$

where the delays are independent based upon the time obtained from scheduling approximation:

$$
t_{c a 1}+t_{s c 1}<t_{c a 2}+t_{s c 2}<\ldots<t_{c a m}+t_{s c m}<T
$$

Now from the derivative of a candidate Lyapunov function is expressed as:

$$
\Delta u(k)=V(k+1)-V(k)
$$

and the related Lyapunov function is:

$$
V(k)=X(k)^{T} P X(k)
$$

each of the fuzzy rules is given as an expression of local delays from current condition from plant towards controller, and vice versa.

$$
\left[\begin{array}{c}
x_{c} \\
x_{p}
\end{array}\right]=\left[\begin{array}{c}
x_{c}(k) \\
x_{c}\left(k-t_{c a 1}\right) \\
x_{c}\left(k-t_{c a 2}\right) \\
\vdots \\
x_{c}\left(k-t_{c a m}\right) \\
x_{p}(k) \\
x_{p}\left(k-t_{s c 1}\right) \\
x_{p}\left(k-t_{s c 2}\right) \\
x_{p}\left(k-t_{s c 3}\right) \\
\vdots \\
x_{p}\left(k-t_{s c m}\right)
\end{array}\right]
$$

For each rule, there is a delay related to a particular condition to the plant and controller. Each of the rules maybe updated through learning procedure or LMI process. Each of the rules is unique on every specific time. In this case, these are associated to a particular relationship of last equation. In terms of the Lyapunov Candidate, this is expressed as in eqn 15 which is consistent to eqn. 8.

$$
\begin{gathered}
V(k+1)-V(k)=\left[\begin{array}{c}
x_{c}(k+1) \\
x_{p}(k+1)
\end{array}\right]^{T} P\left[\begin{array}{l}
x_{c}(k+1) \\
x_{p}(k+1)
\end{array}\right]-\left[\begin{array}{l}
x_{c}(k) \\
x_{p}(k)
\end{array}\right]^{T} P\left[\begin{array}{l}
x_{c}(k) \\
x_{p}(k)
\end{array}\right] \\
V(k+1)-V(k)=\left[\begin{array}{c}
\sum_{j=2}^{m} \sum_{i=2}^{m}\left(h_{j} h_{i}\left(B_{j}^{p}\left(x_{c}\left(k-t_{c a j}\right)\right)\right)+h_{j} A_{j}^{p} x_{p}(k)\right) \\
\sum_{j=2}^{m} \sum_{i=2}^{m}\left(h_{j} h_{i}\left(F_{j}^{c}\left(x_{p}\left(k-t_{s c j}\right)\right)\right)\right)
\end{array}\right]^{T} P
\end{gathered}
$$




$$
\left[\begin{array}{c}
\sum_{j=2}^{m} \sum_{i=2}^{m}\left(h_{j} h_{i}\left(B_{j}^{p}\left(x_{c}\left(k-t_{c a j}\right)\right)\right)+h_{j} A_{j}^{p} x_{p}(k)\right) \\
\sum_{j=2}^{m} \sum_{i=2}^{m}\left(h_{j} h_{i}\left(F_{j}^{c}\left(c_{p}^{i} x_{p}\left(k-t_{s c j}\right)\right)\right)\right)
\end{array}\right]^{T}-\left[\begin{array}{c}
x_{c}(k) \\
x_{p}(k)
\end{array}\right]^{T} P\left[\begin{array}{c}
x_{c}(k) \\
x_{p}(k)
\end{array}\right]
$$

Therefore:

$$
\begin{gathered}
V(k+1)-V(k)=\left[\begin{array}{l}
x_{c}(k+1) \\
x_{p}(k+1)
\end{array}\right]^{T} P\left[\begin{array}{l}
x_{c}(k+1) \\
x_{p}(k+1)
\end{array}\right]- \\
{\left[\begin{array}{c}
x_{c}(k) \\
x_{c}\left(k-t_{c a 1}\right) \\
x_{c}\left(k-t_{c a 2}\right) \\
\vdots \\
x_{c}\left(k-t_{c a m}\right) \\
x_{p}(k) \\
x_{p}\left(k-t_{s c 1}\right) \\
x_{p}\left(k-t_{s c 2}\right) \\
x_{p}\left(k-t_{s c 3}\right) \\
\vdots \\
x_{p}\left(k-t_{s c m}\right)
\end{array}\right]^{T}\left[\begin{array}{c}
x_{c}(k) \\
x_{c}\left(k-t_{c a 1}\right) \\
x_{c}\left(k-t_{c a 2}\right) \\
\vdots \\
x_{c}\left(k-t_{c a m}\right) \\
x_{p}(k) \\
x_{p}\left(k-t_{s c 1}\right) \\
x_{p}\left(k-t_{s c 2}\right) \\
x_{p}\left(k-t_{s c 3}\right) \\
\vdots \\
x_{p}\left(k-t_{s c m}\right)
\end{array}\right]}
\end{gathered}
$$

$t_{c a j}$ and $t_{s c j}$ are the related time delays. Considering the fuzzy system representation:

$$
\begin{aligned}
& V(k+1)-V(k)=\left[\begin{array}{c}
\sum_{j=2}^{m} \sum_{i=2}^{m}\left(h_{j} h_{i}\left(B_{j}^{p}\left(c_{c}^{i} x_{c}\left(k-t_{c a j}\right)\right)\right)+h_{i} A_{i}^{p} x_{p}(k)\right) \\
\sum_{j=2}^{m} \sum_{i=2}^{m}\left(h_{j} h_{i}\left(F_{j}^{c}\left(c_{p}^{i} x_{p}\left(k-t_{s c j}\right)\right)\right)\right)
\end{array}\right]^{T} P \\
& {\left[\begin{array}{c}
\sum_{j=2}^{m} \sum_{i=2}^{m}\left(h_{j} h_{i}\left(B_{j}^{p}\left(c_{c}^{i} x_{c}\left(k-t_{c a j}\right)\right)\right)+h_{i} A_{i}^{p} x_{p}(k)\right) \\
\sum_{j=2}^{m} \sum_{i=2}^{m}\left(h_{j} h_{i}\left(F_{j}^{c}\left(c_{p}^{i} x_{p}\left(k-t_{s c j}\right)\right)\right)\right)
\end{array}\right]-} \\
& {\left[\begin{array}{c}
x_{c}(k) \\
x_{c}\left(k-t_{c a 1}\right) \\
x_{c}\left(k-t_{c a 2}\right) \\
\vdots \\
x_{c}\left(k-t_{c a m}\right) \\
x_{p}(k) \\
x_{p}\left(k-t_{s c 1}\right) \\
x_{p}\left(k-t_{s c 2}\right) \\
x_{p}\left(k-t_{s c 3}\right) \\
\vdots \\
x_{p}\left(k-t_{s c m}\right)
\end{array}\right]^{T} P\left[\begin{array}{c}
x_{c}(k) \\
x_{c}\left(k-t_{c a 1}\right) \\
x_{c}\left(k-t_{c a 2}\right) \\
\vdots \\
x_{c}\left(k-t_{c a m}\right) \\
x_{p}(k) \\
x_{p}\left(k-t_{s c 1}\right) \\
x_{p}\left(k-t_{s c 2}\right) \\
x_{p}\left(k-t_{s c 3}\right) \\
\vdots \\
x_{p}\left(k-t_{s c m}\right)
\end{array}\right]}
\end{aligned}
$$

If only one of the time delays is considered: 


$$
0>\left[\begin{array}{c}
x_{c}(k+1) \\
x_{p}(k+1)
\end{array}\right]^{T} P\left[\begin{array}{c}
x_{c}(k+1) \\
x_{p}(k+1)
\end{array}\right]-\left[\begin{array}{c}
x_{c}(k) \\
x_{c}\left(k-t_{c a j}\right) \\
x_{p}(k) \\
x_{p}\left(k-t_{s c j}\right)
\end{array}\right] P\left[\begin{array}{c}
x_{c}(k) \\
x_{c}\left(k-t_{c a j}\right) \\
x_{p}(k) \\
x_{p}\left(k-t_{s c j}\right)
\end{array}\right]
$$

In here every time delay is local, independent and bounded according to dynamic scheduling algorithm which is based upon the structural codesign section.

\section{Structural Codesign}

The codesign proposal follows the iteration between schedulability and stability analysis following online approximation.

In fact, according to dynamic scheduling algorithm proposal which is based upon structural codesign strategy, these time delays can be seen like a phase modification within the communication period from the involved processes. This scenario presents a complete phase modification at the entire system. The communication network plays a key role in order to define the behavior of the dynamic system in terms of time variance giving a nonlinear behavior. In order to understand such a nonlinear behavior, time delays are incorporated by the use of real-time system theory that allows time delays to be bounded even in the case of causal modifications due to external effects, based upon Priority Exchange [4].

This algorithm bounds Time delays through a real-time scheduling algorithm within communication network. According to Fig. 3, structural reconfiguration takes place as a result of Priority Exchange Scheduling algorithm and the associated user request. This reconfiguration causes a control law modification [19] which is the actual control law reconfiguration.

Scheduling approach potentially modifies frequency execution and communication of tasks in order to give certain priority to some of them during a bounded time as shown in Fig. 3. Furthermore, in this kind of strategy Tasks modifies their priority, it does not imply that neither the period nor the consumption times are modified. Therefore the tasks would have a bounded delay within the sampling time which is reflected as changing on the phase.

Potential modifications onto scheduling approach deploy change in the priorities that affects time delays and the respective control law. The delays are measured as $\Delta t$ and bounded into the inherent control period of time according to eqn. 11. Now by taking partial results from scheduling algorithm like $t_{s j}$ and the related $\Delta t$, the actual time delays are used at the control law for parameters design. The involved time delays are depicted as $\tau_{j}^{i}$ and come from this scheduling design. Other delays like actuators and control delays are not used in the design of the control law, although play an important role. Therefore scheduling and control analysis merge together when time delays are complete bounded even in the case of time variance. The main restriction is in terms of predictable time delays.

The objective here is to present a reconfiguration control strategy developed from the time delay knowledge, following scheduling approximation where time delays are known and bounded according to used scheduling algorithm. The scheduling strategy proposed here pursues to tackle local faults in terms of fault tolerance. In this situation, current time delays would be inevitable. Classical Earliest Deadline First (EDF) plus Priority Exchange (PE) [4] algorithm are used here to decompose time lines and the respective time delays when present. For instance, time delays are supervised for a number of tasks as follows:

$$
C 1 \rightarrow C n T 1 \rightarrow T n
$$


Priority is given as the well-known EDF algorithm, which establishes that the process with the closest deadline has the most important priority [12]. However, when an aperiodic task appears, it is necessary to deploy other algorithms to cope with concurrent conditions. To do so, the PE algorithm is used to manage spare time from the EDF algorithm. The PE algorithm [6] uses a virtual server that deploys a periodic task with the highest priority in order to provide enough computing resources for aperiodic tasks. This simple procedure gives a proximity, deterministic, and dynamic behavior within the group of included processes. In this case, time delays can be deterministic and bounded. As an example, consider a group of tasks as shown in Table 1. In this case, consumption times as well as periods are given in terms of integer units. Remember: the server task is the time given for an aperiodic task to take place on the system.

\begin{tabular}{|l|l|l|}
\hline Name & Consumption (in units) & Period (in units) \\
\hline Task 1 & 2 & 9 \\
\hline Task 2 & 1 & 9 \\
\hline Task 3 & 2 & 10 \\
\hline Server & 1 & 6 \\
\hline
\end{tabular}

Table 1: First example for PE algorithm.

The result of the ordering based upon PE is presented in Fig. 2.

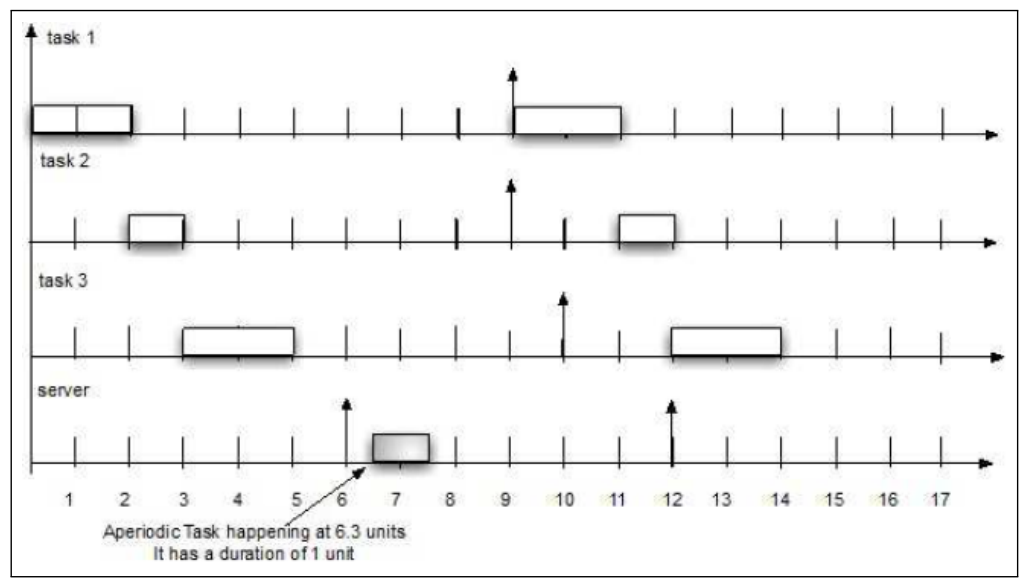

Figure 2: Related organization for PE of tasks in Table 1.

Based on this dynamic scheduling algorithm, time delays are given as current calculations in terms of task ordering. In this case, every time that the scheduling algorithm takes place, the global time delays are modified in the short and long term. For instance, consider the following example, in which four tasks are set, and two aperiodic tasks take place at different times, giving different events with different time delays.

The following task ordering is shown in Fig. 3, using the PE algorithm, where clearly time delays appear.

Now, from this, a resulting ordering of different tiny time delays is given for two scenarios, as shown in Fig. 4.

These two scenarios present two different local time delays that need to be taken into account before hand, in order to settle the related delays according to scheduling approach and control design. These time delays can be expressed in terms of local relations between both dynamical systems. These relations are the actual and possible delays, bounded as marked limits of possible and current scenarios. Then, delays may be expressed as local summations with a high degree 


\begin{tabular}{|l|l|l|}
\hline Name & Consumption (in units) & Period (in units) \\
\hline Task 1 & 2 & 9 \\
\hline Task 2 & 1 & 9 \\
\hline Task 3 & 2 & 10 \\
\hline Server & 1 & 6 \\
\hline Aperiodic task 1 (ap1) & 0.9 & It occurs at 9 \\
\hline Aperiodic task 2 (ap2) & 1.0 & It occurs at 13 \\
\hline
\end{tabular}

Table 2: Second example of PE.

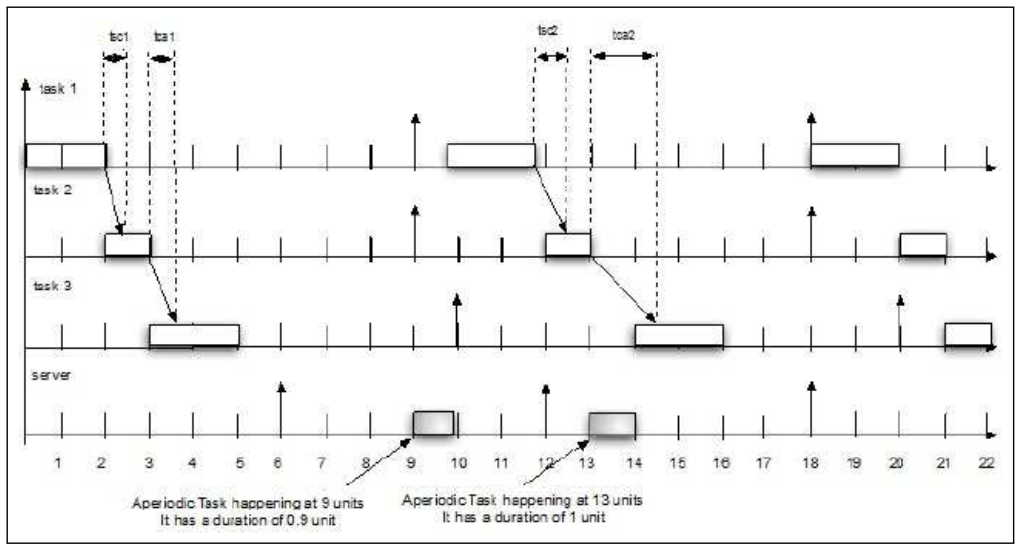

Figure 3: Related time delays are depicted according to both scenarios.

of certainty (as presented in [13]). In this last example, during the second scenario, a total delay is given as:

Total delay $=$ consumption_time_delay_aperiodic_task1 + consumption_time_delay_task1 + tsc $2+$ consumption_time_delay_task $2+$ consumption_time_delay_aperiodic_task $2+$ consumption_time_delay_task3

Now, from this example, $l_{p}$ is equal to 2 and $l_{c}$ is equal to $3 . l_{p}$ and $l_{c}$ are the total number of local delays within one scenario from sensor to control and from control to actuator respectively.

In this case, local time delays as presented in the general eqn. 14 are the result of the iteration of scheduling algorithm. In the approximation presented in this paper the local delays are around four time delays as expressed as last expression called total delays.

The approach followed at the control reconfiguration does not take into account scheduler decision in a direct manner. It takes the time delays as bounded values already defined and used to design a suitable control law. Therefore, according to current state plant values, the related fuzzy rule is selected.

For a NCS, the communication network strongly affects the dynamics of the system, expressed as a time variance that exposes a nonlinear behaviour. Such nonlinearity is addressed by incorporating time delays. From real-time system theory, it is known that time delays are bounded even in the case of causal modifications due to external effects. 


\section{Case of Study}

The case of study consists of a simulation from magnetic levitation system whose sensors and actuators are operated by a "host", the signals from the sensors are sent by the host through a ETHERNET 10/100 network and received by a "server" where the control input is calculated and sent over ETHERNET network to the host. Fig. 5 shows current system configuration as in real state.

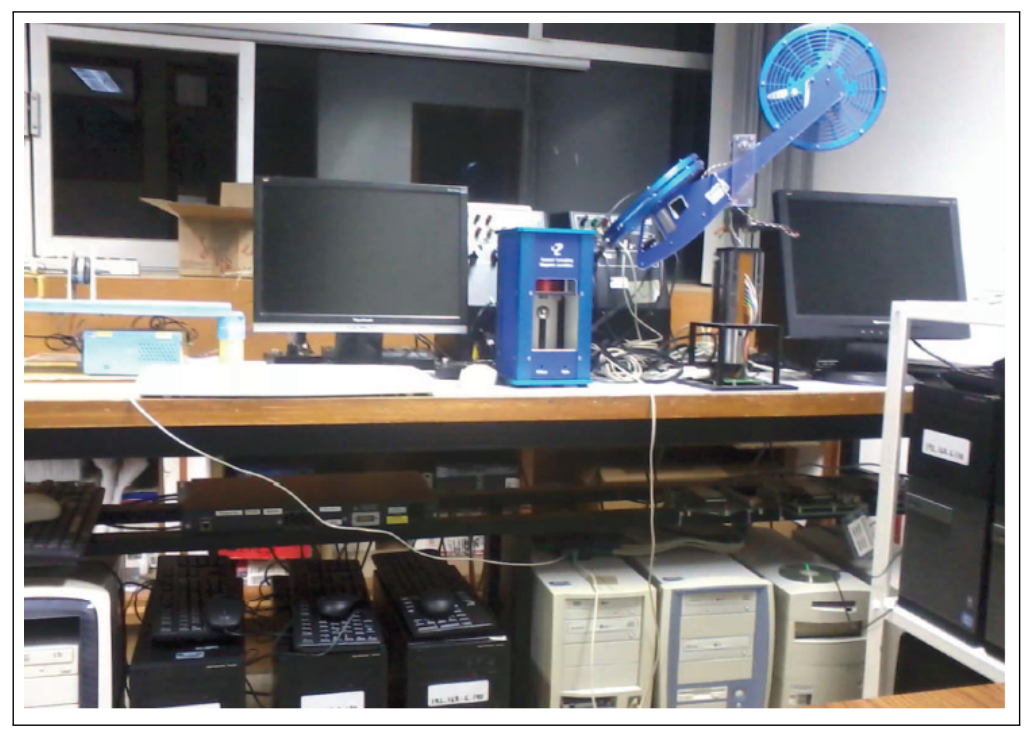

Figure 4: Current configuration of magnetic levitation system.

The system consists of a coil inside a cabin, the coil levitates a steel ball that rests on a black post. The elevation of the ball is measured from the post using a light sensor inside the post. The issue of the experiment is to design a controller that does levitate the steel ball following a desired trajectory.

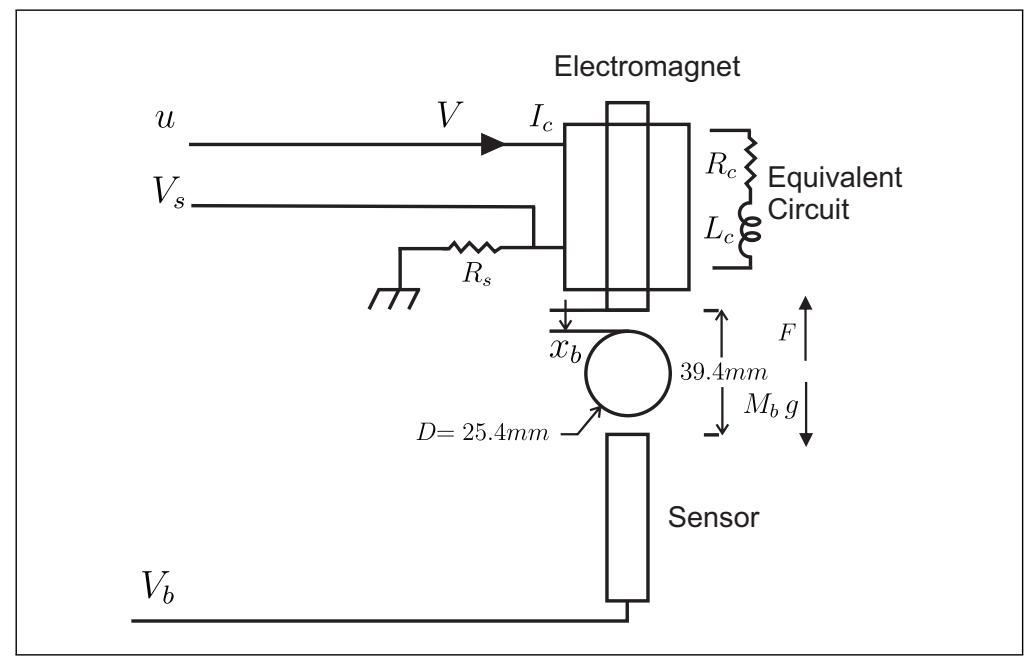

Figure 5: Maglev system.

The nonlinear equations for the Magnetic Levitation System are: 


$$
\begin{aligned}
& \dot{x_{1}}=x_{2} \\
& \dot{x_{2}}=\frac{-K_{m} x_{3}^{2}}{2 M_{b}\left(x_{1}\right)^{2}}+g \\
& \dot{x_{3}}=\frac{1}{L_{c}}\left(-R x_{3}+u\right)
\end{aligned}
$$

were $R=R_{c}+R_{s}$ and $u=V_{c}$ input voltage and

$R_{c}$ electromagnet resistance

$R_{s}$ resistor in series wiht the coil

$K_{m}$ constant of electromagnet force

$M_{b}$ mass of the ball

$g$ gravitacional constant

$L_{c}$ coil inductance

The values of the parameters are provided in [16].

The method generated three rules for the nominal fuzzy control and the range of delay was divided in six parts then the delayed fuzzy control has six fuzzy rules. For the fuzzy model three feedback vector $F_{j}$ were designed to ensures the stability of the overall system.

Following eqn. 10 and resolving eqn. 20 through LMI it is possible to verify the stability in an asymptotic procedure.

\section{Results}

Once the fuzzy control laws are designed according to equations (17)-(20) where the objective is to find a common positive definite matrix $P$ satisfying the linear matrix inequality. Two tests are performed to prove the effectiveness of the method proposed. In all tests the reference trajectory signal applied is a sine signal to be followed by the steel ball.

Three fuzzy rules are defined to approximate the magnetic levitation system by means of three linear models, as follows:

Rule 1:

IF $x_{1}(t)$ is about $0.006 \mathrm{~m}$,

THEN $x(k+1)=A_{1} x(k)+B_{1} u(k)$

Rule 2:

IF $x_{1}(t)$ is about $0.009 \mathrm{~m}$,

THEN $x(k+1)=A_{2} x(k)+B_{2} u(k)$

Rule 3:

IF $x_{1}(t)$ is about $0.013 m$,

THEN $x(k+1)=A_{3} x(k)+B_{3} u(k)$

where $x_{1}$ is the ball position in meters and 


$$
\begin{aligned}
A_{1} & =\left[\begin{array}{ccc}
1.0016 & 0.0010 & 0 \\
3.2718 & 1.0016 & -0.055 \\
0 & 0 & 0.9737
\end{array}\right] \\
A_{2} & =\left[\begin{array}{ccc}
1.0011 & 0.0010 & 0 \\
2.1808 & 1.0011 & -0.0175 \\
0 & 0 & 0.9737
\end{array}\right] \\
A_{3} & =\left[\begin{array}{ccc}
1.0012 & 0.0010 & 0 \\
2.3774 & 1.0012 & -0.0212 \\
0 & 0 & 0.9737
\end{array}\right] \\
B_{1}=B_{2}=B_{3} & =\left[\begin{array}{c}
0 \\
0 \\
0.0024
\end{array}\right]
\end{aligned}
$$

The control gains obtained by means LMI Matlab's toolbox are:

$$
\begin{aligned}
& F_{1}=\left[\begin{array}{lll}
-51650 & -1102 & 379
\end{array}\right] \\
& F_{2}=\left[\begin{array}{lll}
-48530 & -1058 & 341
\end{array}\right] \\
& F_{3}=\left[\begin{array}{lll}
22546 & -479 & 128
\end{array}\right]
\end{aligned}
$$

These control gain values guarantee the stability of the system during the presence of local time delays according to table 3 . In this case local time delays are responsive in terms on an periodic external task, that is presented every determined seconds.

With the next positive definite matrix $P$ :

$$
P=\left[\begin{array}{ccc}
0.1980 & 0.0042 & -0.0007 \\
0.0042 & 0.0001 & -0.0000 \\
-0.0007 & -0.0000 & 0.0000
\end{array}\right]
$$

In order to prove the effectiveness of the metod proposed, two experiments were performed, in the first scenario the plant tracks a reference signal (sine wave) and the transmission task were the following (Table 3)

\begin{tabular}{|l|l|l|}
\hline Name & Consumption (in milliseconds) & Period (in milliseconds) \\
\hline Task 1 & 2 & 10 \\
\hline Task 2 & 1 & 12 \\
\hline Task 3 & 2 & 14 \\
\hline Aperiodic Task & 1 & 90 \\
\hline
\end{tabular}

Table 3: PE

The activation task was performed using Stateflow as shown in Fig. 6 where according to Table 3 the task 1 is the controller transmission task and has the priority one, the task 2 is the sensor transmission task an has the priority two and the task 3 and 4 are the transmissions task 
and the sporadic transmission task from others nodes.

The system response obtained in this first experiment is shown in Fig. 7 (without time delays).

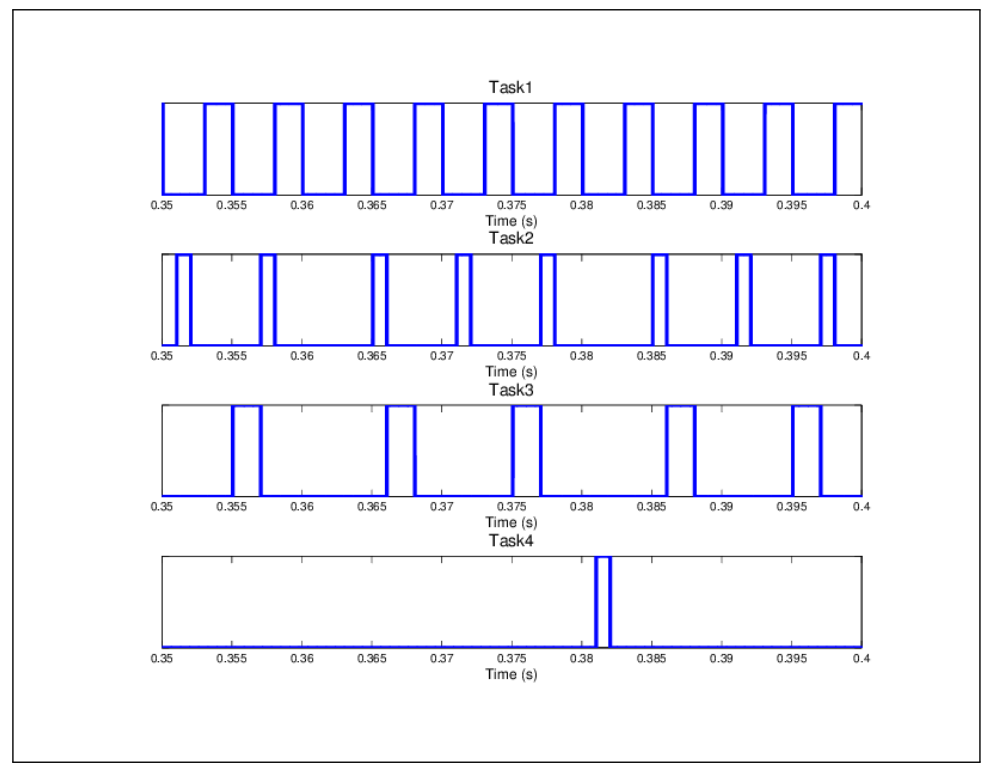

Figure 6: Activation tasks following table 3

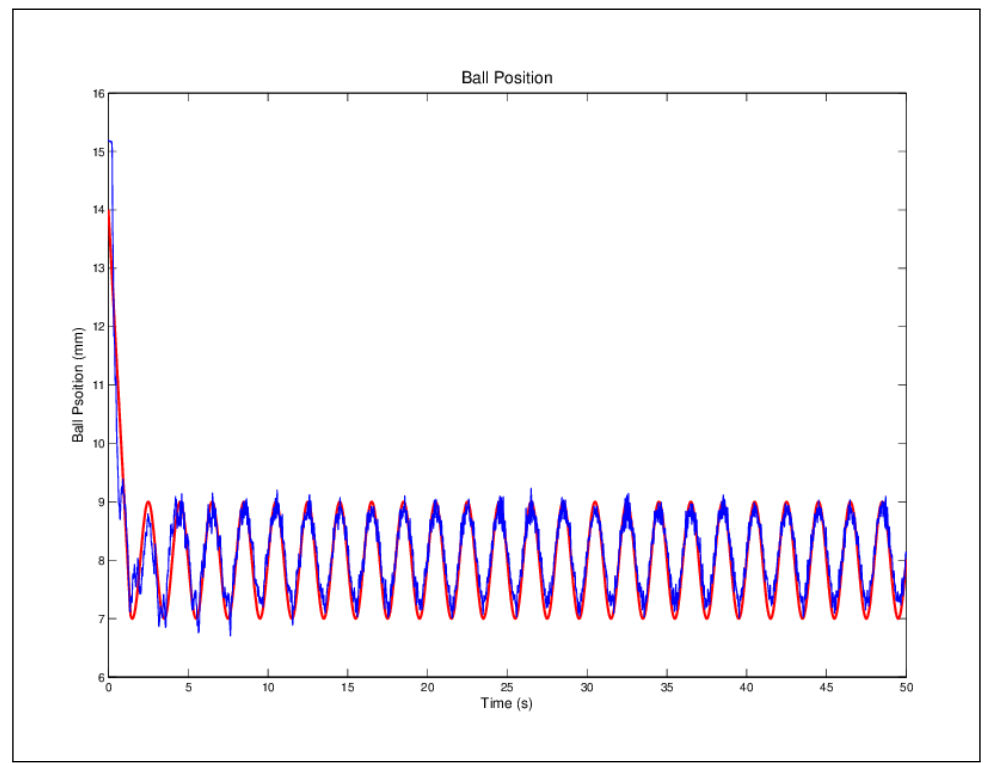

Figure 7: Ball Position Response in the first scenario

The second test is to apply a time delay less than the sampling period according to the total time delay. Fig. 8 shows the behavior of the system which maintains stability with a delay of 2 $\mathrm{ms}$ and a sampling period of $90 \mathrm{~ms}$ related to the aperiodic task. The behavior is very similar to the system without time delay. 


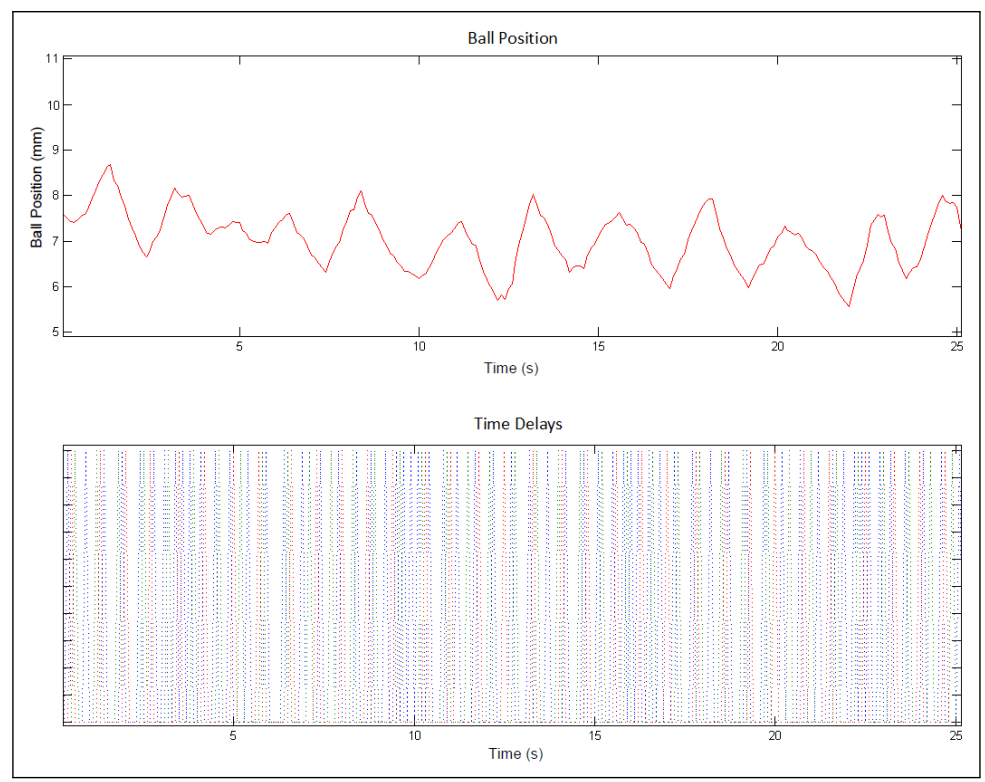

Figure 8: Ball Position Response in the second scenario

\section{Conclusion}

It has been established a supervisory fuzzy control to minimize the effects caused by the time delay due to communication into the network which is designed through codesign strategy. This approach introduces the time delay produced by scheduling approach named Priority Exchange Procedure. With this fuzzy model a fuzzy control is designed and the stability analysis is proposed for this controller. This approach shows that the system with a time delay smaller than sampling period but with a complex behaviour maintains the stability, the stability analysis for time varying delay and a bound for this delay remain a work in the future.

Although the example related to the time delays is fairly demonstrative it becomes challenging in terms of the dynamic scheduling approach where local time delays is pursued according to eqn. 10 in a general form and implemented through state flow tool in each node.

\section{Acknowledgments}

The authors acknowledge the support of UNAM-PAPIIT IN100813, CONACYT 176556 and PICCO 10-53.

\section{Bibliography}

[1] Almeida, L. et al (2002); The FTT-CAN protocol: why and how. IEEE Transactions on Industrial Electronics, 49(6):1189-1201.

[2] Benítez-Pérez, H. et al (2013); Networked Control Systems Design considering Scheduling Restrictions and Local Faults using Local State Estimation, International Journal of Innovative Computing, Information and Control (IJICIC), 9(8): 3225-3239. 
[3] Benítez-Pérez, H. et al (2012); Networked Control Systems design considering scheduling restrictions, International Journal on Advanced Fuzzy Systems, http://dx.doi.org/10.1155/2012/927878.

[4] Benítez-Pérez, H. et al (2012); Networked Control Systems Design considering Scheduling Restrictions and Local Faults, International Journal of Innovative Computing, Information and Control (IJICIC), 8(10):8515-8526.

[5] Benítez-Pérez, H.; García-Nocetti, F. (2005); Reconfigurable Distributed Control, Springer.

[6] Buttazo, G. (2004); Hard Real-Time Computing Systems, Springer.

[7] Czornik, A.; Swierniak, A. (2003); On Direct Controllability of Discrete Time Jump Linear System, Tech. Rep., Mathematical Biosciences Institute, The Ohio State University.

[8] Eidson, J.C. et. al (2002); IEEE-1588 Standard for a Precision Clock Synchronization Protocol for Networked Measurement and Control Systems, IEEE Standard, 1588-2002.

[9] Fridman, E.; Shaked, U. (2003); Delay-dependent stability and $\mathrm{H} \infty$ control: constant and time-varying delays, International Journal Control, 76(1):48-60.

[10] Lian, F. et al (2002); Network Design Consideration for Distributed Control Systems, IEEE Transactions on Control Systems Technology, 10(2):297-307.

[11] Liu, J. (2000); Real-Time Systems, Prentice Hall.

[12] Méndez-Monroy, P.E.; Benítez-Pérez, H. (2009); Supervisory Fuzzy Control for Networked Control Systems, International Journal Innovative Computing, Information and Control Express Letters, ICIC-EL, 3-2, 233-240.

[13] Méndez-Monroy, P.E.; Benítez-Pérez, H. (2011); Codesign Strategy Based Upon Fuzzy Control for Networked Control Systems and a Scheduling Algorithm, IEEE International Conference on Networking, Sensing and Control (ICNSC), 221-226.

[14] Moarref, M.; Rodrigues, L. (2015); Piecewise Affine Networked Control Systems, IEEE Transactions and Control of Network Systems, DOI 10.1109/TCNS.2015.2428452.

[15] Nilsson, J. (1998); Real-Time Control Systems with Delays, Ph.D. Thesis, Lund Institute of Technology, Dept. of Automatic Control.

[16] Quanser Inc.(2006), Magnetic Levitation Experiment, Quanser Consulting.

[17] Tzes, A. et. al (2005); Development and Experimental Verification of a Mobile Client-Centric Networked Controlled System, European Journal of Control, 11(3): 229-241.

[18] Walsh, G. C. et. al (1999); Stability Analysis of Networked Control Systems, American Control Conference, 2876-2880.

[19] Wang, Y.; Sun, Z. (2007); H-inf Control of Networked Control Systems Via LMI Approach, International Journal of Innovative Computing, Information and Control, 3(2):343-352.

[20] Xiao, L. et al (2000); Control with Random Communication Delays via a Discrete-Time Jump System Approach, American Control Conference, 3:2199-2204. 
[21] Yu, M. et al (2003); An LMI Approach to Networked Control Systems with Data Packet Dropout and Transmission Delays, International Journal of Hybrid Systems, 3(2):3545 3550 .

[22] Zhang, W. (2001); Stability Analysis of Networked Control Systems. Ph.D. thesis, Case Western Reserve University, Dept. of Electrical Engineering and Computer Science.

[23] Zhu, X. et al (2008); State Feedback Control Design of Networked Control Systems with Time Delay in the Plant, International Journal of Innovative Computing, Information and Control, 4(2): 283-290.

[24] http://www.mathworks.com/help/robust/lmis.html (retrieved on July 20, 2015). 